\title{
Sex, age, and hospitalization drive antibody responses in a COVID-19 convalescent plasma donor population
}

\author{
Sabra L. Klein, ${ }^{1,2,3}$ Andrew Pekosz, ${ }^{1,4}$ Han-Sol Park, ${ }^{1}$ Rebecca L. Ursin, ${ }^{2}$ Janna R. Shapiro, ${ }^{3}$ Sarah E. Benner, ${ }^{5}$ Kirsten Littlefield, ${ }^{1}$ \\ Swetha Kumar, ${ }^{6}$ Harnish Mukesh Naik, ${ }^{6}$ Michael J. Betenbaugh, ${ }^{6}$ Ruchee Shrestha, ${ }^{5}$ Annie A. Wu, ${ }^{5}$ Robert M. Hughes, ${ }^{5}$ \\ Imani Burgess, ${ }^{5}$ Patricio Caturegli, ${ }^{5}$ Oliver Laeyendecker, ${ }^{7,8}$ Thomas C. Quinn, ${ }^{7,8}$ David Sullivan, ${ }^{1}$ Shmuel Shoham, \\ Andrew D. Redd, ${ }^{7,8}$ Evan M. Bloch, ${ }^{5}$ Arturo Casadevall, ${ }^{1}$ and Aaron A.R. Tobian ${ }^{5}$ \\ ${ }^{1}$ W. Harry Feinstone Department of Molecular Microbiology and Immunology, ${ }^{2}$ Department of Biochemistry and Molecular Biology, ${ }^{3}$ Department of International Health, and ${ }^{4}$ Department of Environmental \\ Health and Engineering, Johns Hopkins Bloomberg School of Public Health, Baltimore, Maryland, USA. 'Department of Pathology, Johns Hopkins School of Medicine, Baltimore, Maryland, USA. \\ ${ }^{6}$ Advanced Mammalian Biomanufacturing Innovation Center, Department of Chemical and Biomolecular Engineering, Johns Hopkins University, Baltimore, Maryland, USA. ${ }^{7}$ Department of Medicine, \\ Division of Infectious Diseases, Johns Hopkins School of Medicine, Baltimore, Maryland, USA. ${ }^{8}$ Division of Intramural Research, National Institute of Allergy and Infectious Diseases (NIAID), \\ NIH, Bethesda, Maryland, USA.
}

Convalescent plasma is a leading treatment for coronavirus disease 2019 (COVID-19), but there is a paucity of data identifying its therapeutic efficacy. Among 126 potential convalescent plasma donors, the humoral immune response was evaluated using a severe acute respiratory syndrome coronavirus 2 (SARS-CoV-2) virus neutralization assay with Vero-E6-TMPRSS2 cells; a commercial IgG and IgA ELISA to detect the spike (S) protein S1 domain (EUROIMMUN); IgA, IgG, and IgM indirect ELISAs to detect the full-length S protein or S receptor-binding domain (S-RBD); and an IgC avidity assay. We used multiple linear regression and predictive models to assess the correlations between antibody responses and demographic and clinical characteristics. IgC titers were greater than either IgM or IgA titers for S1, full-length S, and S-RBD in the overall population. Of the 126 plasma samples, $101(80 \%)$ had detectable neutralizing antibody (nAb) titers. Using nAb titers as the reference, the IgC ELISAs confirmed $95 \%-98 \%$ of the nAb-positive samples, but $20 \%-32 \%$ of the nAb-negative samples were still IgC ELISA positive. Male sex, older age, and hospitalization for COVID-19 were associated with increased antibody responses across the serological assays. There was substantial heterogeneity in the antibody response among potential convalescent plasma donors, but sex, age, and hospitalization emerged as factors that can be used to identify individuals with a high likelihood of having strong antiviral antibody responses.

\section{Introduction}

Severe acute respiratory syndrome coronavirus 2 (SARS-CoV-2), the causative agent of coronavirus disease 2019 (COVID-19), emerged in Wuhan, China, in December 2019. Following the rapid, global spread of SARS-CoV-2 in March 2020, COVID-19 was declared a pandemic. By October 2020, over 35 million cases were confirmed, spanning 188 countries or territories and accounting for over 1 million deaths (1). Preventive and treatment options are limited, of which antibody therapy (i.e., convalescent plasma collected from individuals after recovery from COVID-19) has

Authorship note: SLK, EMB, AC, and AART are co-senior authors. Conflict of interest: EMB reports receiving personal fees and nonfinancial support from Terumo BCT and personal fees and nonfinancial support from Grifols Diagnostic Solutions. EMB is a member of the United States FDA Blood Products Advisory Committee. Any views or opinions that are expressed in this manuscript are those of the authors, based on their own scientific expertise and professional judgment; they do not necessarily represent the views of either the Blood Products Advisory Committee or the formal position of the FDA, and also do not bind or otherwise obligate or commit either the advisory committee or the agency to the views expressed. Copyright: (5) 2020, American Society for Clinical Investigation.

Submitted: July 7, 2020; Accepted: August 6, 2020; Published: October 19, 2020

Reference information: J Clin Invest. 2020;130(11):6141-6150.

https://doi.org/10.1172/JCl142004 emerged as a leading treatment for COVID-19 (2). Observational findings are encouraging, suggesting improved clinical outcomes in those who are transfused with COVID-19 convalescent plasma (CCP), including radiological resolution, a reduction in viral loads, and improved survival (3-8). Although 2 randomized trials assessing CCP in China and Europe were terminated early and underpowered, they did not find clinically significant differences between the study arms $(6,9)$. Nonetheless, there is a lack of standardization of units of CCP that are being transfused, in large part because of limited data correlating antibody assays with formal virus neutralization activity.

Antibody responses that target the immunodominant SARSCoV-2 spike (S) protein - specifically, those that target the S protein receptor-binding domain (S-RBD) - are thought to be highly associated with virus neutralization by blocking the interaction between $\mathrm{S}-\mathrm{RBD}$ and the virus receptor angiotensin-converting enzyme 2 (AEC2) (10). The SARS-CoV-2 S protein is a highly glycosylated, trimeric protein that requires proteolytic processing to become fusogenic and mediate virus-host membrane fusion $(11,12)$. The S-RBD domain is partially masked in the prefusion structure of S protein and must be converted to an "open" conformation for optimal binding of S to ACE2 (13). Neutralizing antibodies (nAbs) are of particular interest, because they prevent viral 
Table 1. Demographic data for the convalescent plasma donors

$\begin{array}{lccc} & \text { All } & \text { Females } & \text { Males } \\ \boldsymbol{n}(\%) & 126 & 58(46) & 68(54) \\ \text { Age (yr), median (IQR) } & 42(29-53) & 41.5(28-55) & 42(31.5-53) \\ \text { Age categories (yr), } \boldsymbol{n}(\%) & & & \\ 19-44 & 74(58.7) & 34(58.6) & 40(58.8) \\ 45-64 & 41(32.5) & 19(32.8) & 22(32.4) \\ 65+ & 11(8.7) & 5(8.6) & 6(8.8) \\ \text { Race/ethnicity, } \boldsymbol{n}(\%) & & & \\ \text { White } & 94(74.6) & 42(72.4) & 52(76.5) \\ \text { African American } & 4(3.2) & 1(1.7) & 3(4.4) \\ \text { Asian } & 14(11.1) & 8(13.8) & 6(8.8) \\ \text { Latino } & 5(4) & 2(3.4) & 3(4.4) \\ \text { Mixed/other/unknown } & 9(7.1) & 5(8.6) & 4(5.9) \\ \text { Hospitalized, } \boldsymbol{n} \text { (\%) }{ }^{A} & 11(8.9) & 6(10.7) & 5(7.4) \\ \text { No. of days, median (IQR) } & 5(2-6) & 5(2-5) & 4(3-6) \\ \text { Days since swab collection, median (IQR) } & 43(38-48) & 44.5(39-49) & 41(37-48)\end{array}$

${ }^{A}$ Hospitalization status missing for 2 donors. Percentages were calculated from the total of the available data. quantified as area under the curve (AUC), were greater than titers of either IgM or IgA $(P<0.05$ in each case; Figure 1, C and F). We observed considerable heterogeneity in the antibody responses to either S or S-RBD (Figure 1, D and $G$ ), but the AUC values for both anti-S-IgG and antiS-RBD-IgG were positively associated with the AUC values for anti-S-IgM and -IgA and anti-S-RBD-IgM and -IgA, respectively $(r>0.5$ in each case; Figure $1, \mathrm{E}$ and $\mathrm{H})$. Finally, AUC values for anti-S-IgG, -IgM, and -IgA and anti-S-RBD-IgG, -IgM, and -IgA were strongly correlated with the respective geometric mean titers, with cutoffs based on the negative control samples run on the same plates (Supplemental Figure 1, A-O; supplemental material available online with this article; https://doi. org/10.1172/JCI142004DS1).

Defining functional antibodies in CCP. To assess the functionality of antibodies that recognize SARS-CoV-2 in convalescent plasma, we performed microneutralization and IgG avidity assays. We used the reciprocal plasma dilution providing protection from SARS-CoV-2 to calculate the AUC for the microneutralization assay. Of the 126 plasma samples screened, 101 (80\%) had infection by blocking cell-surface attachment as well as inhibiting host membrane fusion $(14,15)$. Administration of CCP containing these nAbs to individuals with COVID-19 has been shown to result in rapid viral clearance, indicating its functionality as an antiviral agent (6). Non-nAbs also play a key role in viral clearance, as they are needed for antibody-dependent cellular cytotoxicity, antibody-dependent, cell-mediated phagocytosis, and complement activation (16). The contribution of other antibody types such as IgM and IgA to resolution and potential protection from SARS-CoV-2 infection is not clear. Using plasma samples from 126 patients who recovered from COVID-19 following mild or moderate disease, we compared a commercial ELISA, 2-step S protein-directed ELISAs, and microneutralization assays in order to assess how the age and sex of the donor, history of hospitalization for COVID-19, and the time of plasma collection relative to infection could be used to understand the variability of antibody responses to SARS-CoV-2.

\section{Results}

Ig isotyping in CCP. Convalescent plasma was collected from 126 patients with molecular confirmed SARS-CoV-2 infection. The population consisted of more males (54\%) than females (46\%), with a median age of 42 years (IQR 29-53) (Table 1). Most of the patients were classified as having mild to moderate disease, with less than $10 \%$ having been hospitalized with COVID-19. Plasma samples were collected from patients a median of 43 days (IQR 38-48) after an initial $\mathrm{PCR}^{+}$nasal swab test. Plasma samples were used for isotyping antibodies that recognized SARS-CoV-2 S antigens. Using the EUROIMMUN ELISA that recognizes either IgG or IgA against the S protein domain S1, we determined that both isotypes were highly detectable in plasma, with arbitrary unit (AU) values for anti-S1-IgG being greater than those for anti-S1-IgA $(P<0.05$; Figure $1 \mathrm{~A})$ but positively associated with each other $(r$ $>0.5$; Figure 1B). We obtained consistent results with the indirect ELISAs that recognized either S or S-RBD, in which titers of IgG, detectable nAbs (Figure 2A). The avidity assay defines the binding characteristics of IgG; the OD reading in the presence of various concentrations of urea was used to calculate AU for IgG avidity (Figure 2B). We noted a positive correlation between the results from the microneutralization assay and the IgG ELISAs for S1, S, and S-RBD and anti-S1-IgG avidity (Figure 2C), with the correlation of nAbs and S-RBD antibodies being strongest and the anti-S1 $\operatorname{IgG}$ avidity being weakest. Because virus neutralization is currently considered the most critical antibody characteristic associated with potential protection from infection, we assessed the association of the $3 \mathrm{~S}$ protein IgG ELISAs with the microneutralization assay (Figure 2D). We designated negative cutoffs as less than 20 for nAbs, less than 0.8 AU for S1-IgG, and less than 1:320 endpoint titers for $\mathrm{S}$ and S-RBD ELISAs. The overall ability of the IgG assays to confirm positive nAb samples was good, with S1-IgG at $96 \%$, S-IgG at $98 \%$, and S-RBD-IgG at $95 \%$. In contrast, the ability of the IgG assays to confirm negative nAb samples was consistently low, with S1-IgG at 32\%, S-IgG at 20\%, and S-RBD-IgG at $28 \%$. These data suggest that ELISAs may not be superior for confirming samples that do not contain adequate nAb titers.

Host factors contributing to improved antibody responses in CCP. Using the unadjusted AUC values, we determined that males consistently had greater nAb, anti-S-IgG, and anti-S-RBD-IgG responses than did females $(P<0.05$ in each case, Supplemental Table 1). Both nAb and anti-S-RBD-IgG titers, in particular, were consistently higher among males than females within diverse age categories and among nonhospitalized patients (Supplemental Table 1 and Supplemental Figure 2).

We used multiple linear regression models to isolate the effects of sex, age, hospitalization, and time since the $\mathrm{PCR}^{+}$nasal swab on the antibody response to SARS-CoV-2, while adjusting for the other parameters (Supplemental Table 2 and Figure 3). As shown in Figure 3, A-P, and Supplemental Table 2, male sex, older age, and hospitalization for COVID-19 were each associated with greater nAb AUC values, anti-S1-IgG AU, anti-S-IgG AUC values, and anti- 

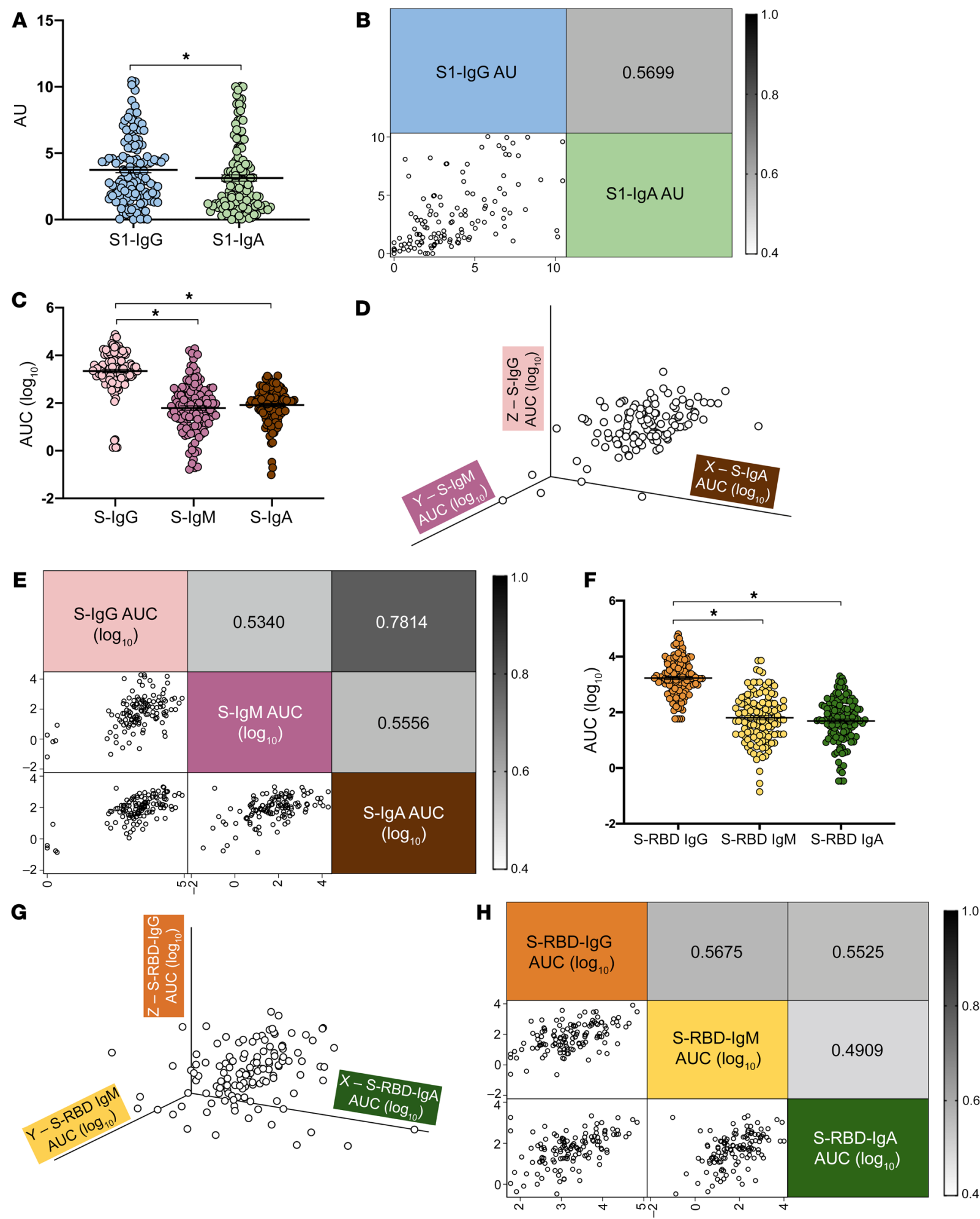

Figure 1. IgG is the primary isotype produced against SARS-CoV-2 S protein. Convalescent plasma samples from patients who recovered from COVID-19 were used to assess antibody isotypes that recognize SARS-CoV-2 antigens. (A) Commercial kits from EUROIMMUN were used to measure total IgG and IgA antibodies against the SARS-CoV-2 S protein domain S1 at an $\mathrm{OD}$ of $450 \mathrm{~nm}\left(\mathrm{OD}_{450}\right)$ and were compared with a calibrator to yield AU. (B) The correlation between anti-S1 isotypes is graphed, with the $r$ value noted. Indirect ELISAs were used to measure IgG, IgM, and IgA antibody levels against $\mathrm{S}$ protein (C) and IgG, IgM, and IgA against S-RBD (F) and are graphed as AUC values. The heterogeneity of the IgG, IgM, and IgA antibody responses against $\mathrm{S}$ protein (D) and S-RBD (C) are shown in 3D scatter plots, with IgA on the $x$ axis, IgM on the $y$ axis, and IgC on the $z$ axis. The correlations between IgG, IgM, and IgA for S protein $(E)$ and S-RBD $(\mathbf{H})$ are included, with $r$ values shown, and are shaded darker for higher correlation values or lighter for lower correlation values. Data indicate the mean \pm SEM. $n=126 .{ }^{*} P<0.05$, by paired $t$ test. 

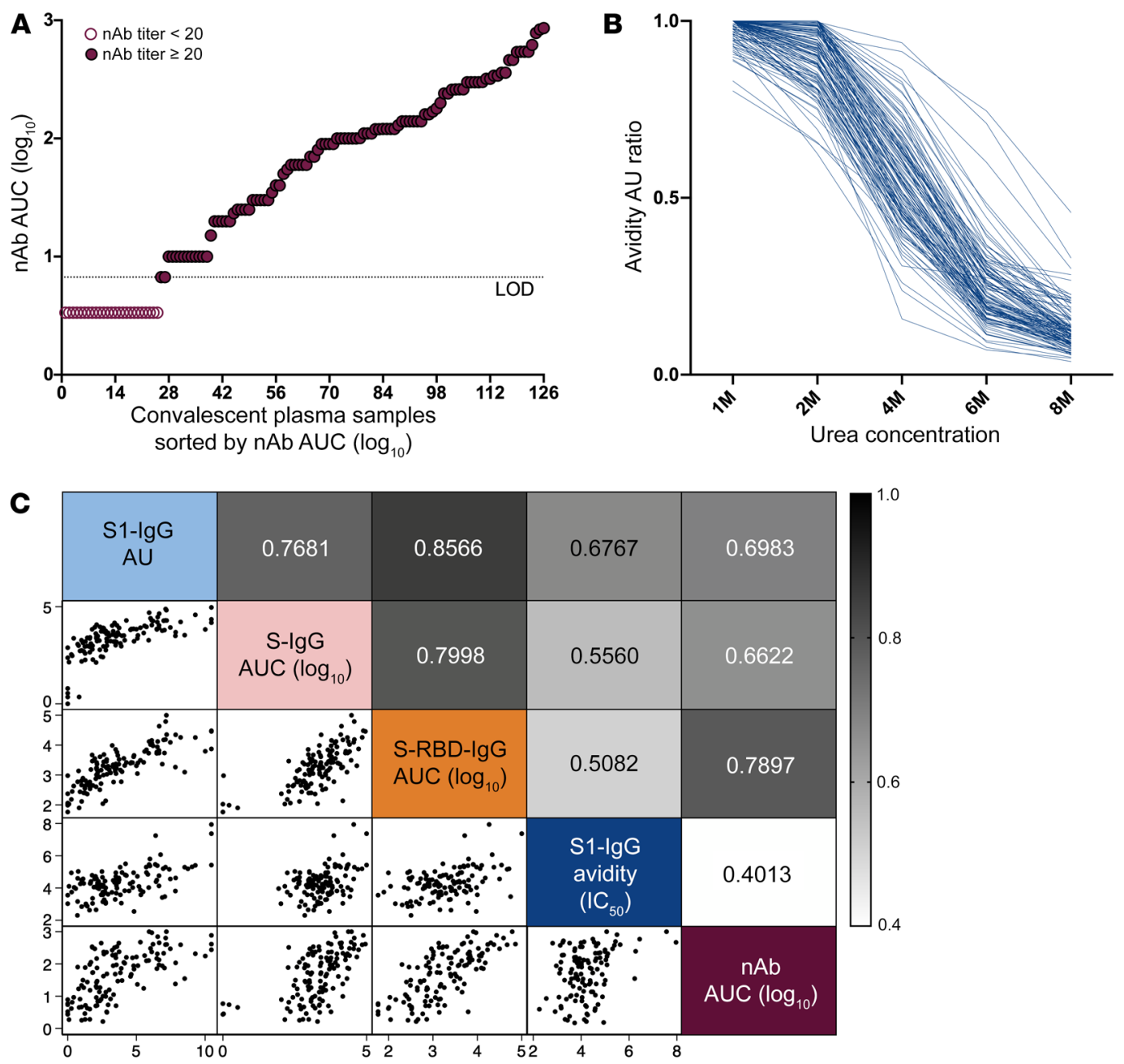

\begin{tabular}{|l|cccc|}
\hline Test & Positive & \% Positive & Negative & \% Negative \#\# \\
\hline nAb titer binary $($ neg $<20)$ & 101 & ref & 25 & ref \\
S1-IgG (neg < AU 0.8) & 114 & 96 & 12 & 32 \\
S-IgG (neg < titer 1:320) & 119 & 98 & 7 & 20 \\
S-RBD-IgG (neg < titer 1:320) & 114 & 95 & 12 & 28 \\
\hline
\end{tabular}

\#Percentage of samples that had positive $n A b$ titers that were also positive byELISA

\#\#ercentage of samples that had negative nAb titers that were also negative by ELISA

Figure 2. $\mathrm{nAb}$ titers correlate with IgG antibodies that recognize SARS-CoV-2 S protein. Convalescent plasma samples from patients recovered from COVID-19 were used to assess functional antibody levels. (A) Microneutralization assays were performed on each plasma sample in 2-fold serial dilutions, with the AUC calculated for all samples with a titer of 20 or higher. (B) Avidity assay used varying amounts of urea to dissociate the anti-S1 spike protein domain IgG/antigen complex from each plasma sample (represented as arbitrary units, $\mathrm{AU}$ ) to identify the optimal avidity $\mathrm{AU}$ ratio (2 M urea) for subsequent analyses. (C) The correlations between nAb AUC values, anti-S1-lgG avidity AU, anti-S1-IgC AU, anti-S-lgG AUC, and anti-S-RBD-IgC AUC are shown, with the $r$ values indicated as well as darker shading for higher correlation values and lighter shading for lower correlation values. (D) For each ELISA assay, the percentage of positive and negative (neg) samples was defined and compared with the nAb AUC (ref), with the negative cutoff values for each assay listed.

RBD-IgG AUC values $(P<0.05$ in each case). When comparing the effect size of each parameter, hospitalization was associated with the largest increase in antibody response (Figure 3Q). A comparison of the 4 assays revealed that male sex, older age, and hospitalization had the largest effect on the anti-S1-IgG response. The only antibody measurement associated with time (days, scaled by 10) since a positive SARS-CoV-2 diagnosis was the $\mathrm{nAb}$ response, which decreased as the time from collection of the diagnostic $\mathrm{PCR}^{+}$ nasal swab increased $(P<0.05$; Figure $3, P$ and $Q)$.
Predictors of strong antibody responses in donors of CCP. The convalescent plasma samples were categorized into quartiles on the basis of their nAb AUC value, anti-S1-IgG AU, anti-SIgG AUC value, or anti-RBD-IgG AUC value, resulting in scores ranging from $\mathrm{O}$ (lowest quartile for each antibody measure) to 12 (highest quartile for each antibody measure) to model the optimal antibody responses in convalescent plasma (Figure 4A and Supplemental Table 3 ). Thirteen percent (16 of 126) of donors were in the lowest decile in all measured responses. Multiple 
O S1-IgG AU OS-IgG AUC $\left(\log _{10}\right) \quad$ O S-RBD-IgG AUC $\left(\log _{10}\right) \quad$ O nAb AUC $\left(\log _{10}\right)$
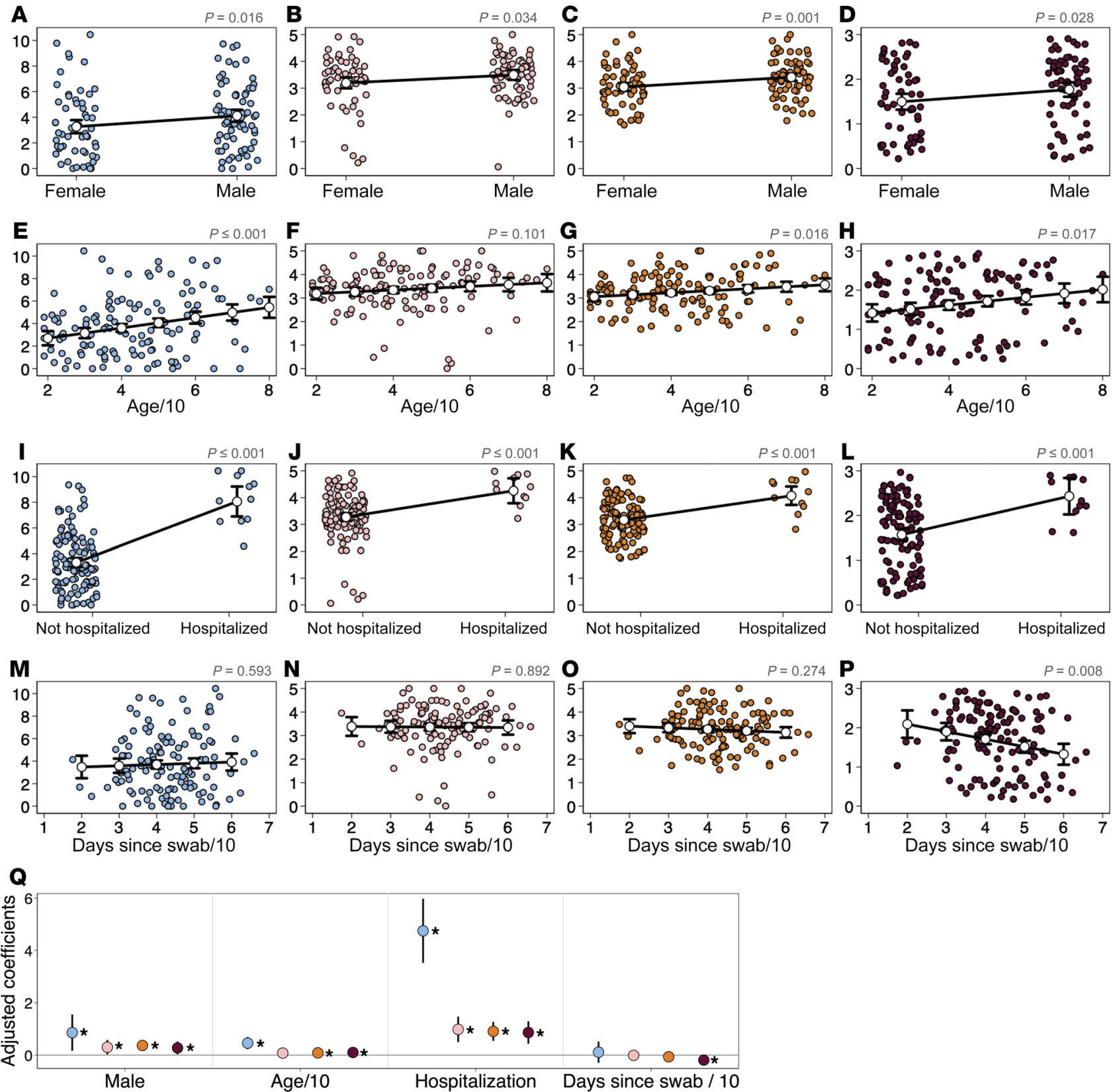

Figure 3. Sex, age, hospitalization, and time since collection of the PCR ${ }^{+}$nasal swab are associated with antibody responses to SARS-CoV-2. Multiple linear regression models were used to analyze the continuous outcomes of anti-S protein domain S1-IgC AU (A, E, I, and $\mathbf{M})$, anti-S-lgG AUC (B, F, J, and $\mathbf{N})$, anti-S-RBD AUC (C, J, K, and $\mathbf{O})$, and nAb (NT) AUC (D, H, L, and $\mathbf{P})$. For each outcome, the model included parameters for the 4 predictors of interest: sex $(\mathbf{A}-\mathbf{D})$, age in decades $(\mathbf{E}-\mathbf{H})$, hospitalization status $(\mathbf{J}-\mathbf{L})$, and number of days since collection of the $\mathrm{PCR}^{+}$nasal swab (M-P). Regression models included the 124 subjects for whom complete predictor data were available (hospitalization status was missing for 2 subjects). In each panel, colored circles show the raw data, and white dots show the marginal effect of the given predictor or the model-predicted outcome (with a 95\% $\mathrm{Cl}$ ) for the average person for different levels of the given predictor. $P$ values at the top of each panel represent the significance level for the parameter. The 4 models are summarized in Q, where the position of the marker indicates the coefficient value and the $95 \% \mathrm{Cl}$, and asterisks indicate significance $\left({ }^{*} P<0.05\right)$.

linear regression analysis of the composite score encompassing the quartiles for each antibody measure revealed that male sex, older age, and hospitalization for severe COVID-19 could each predict greater antibody responses against SARS-CoV-2 (Figure 4, B-D). In contrast, the time elapsed since the diagnostic $\mathrm{PCR}^{+}$nasal swab was not predictive of greater antibody responses (Figure $4 \mathrm{E}$ ). In terms of effect size, we observed an average numerical increase of 1.5 in the quartile score for males compared with that for females, an increase of less than 1 for age (in decades), and an average increase of 5 for hospitalization (Figure 4F). Taken together, these data suggest that hospitalization for severe COVID-19 and male sex could be used as predictors of greater convalescent plasma antibody responses against SARS-CoV-2. 

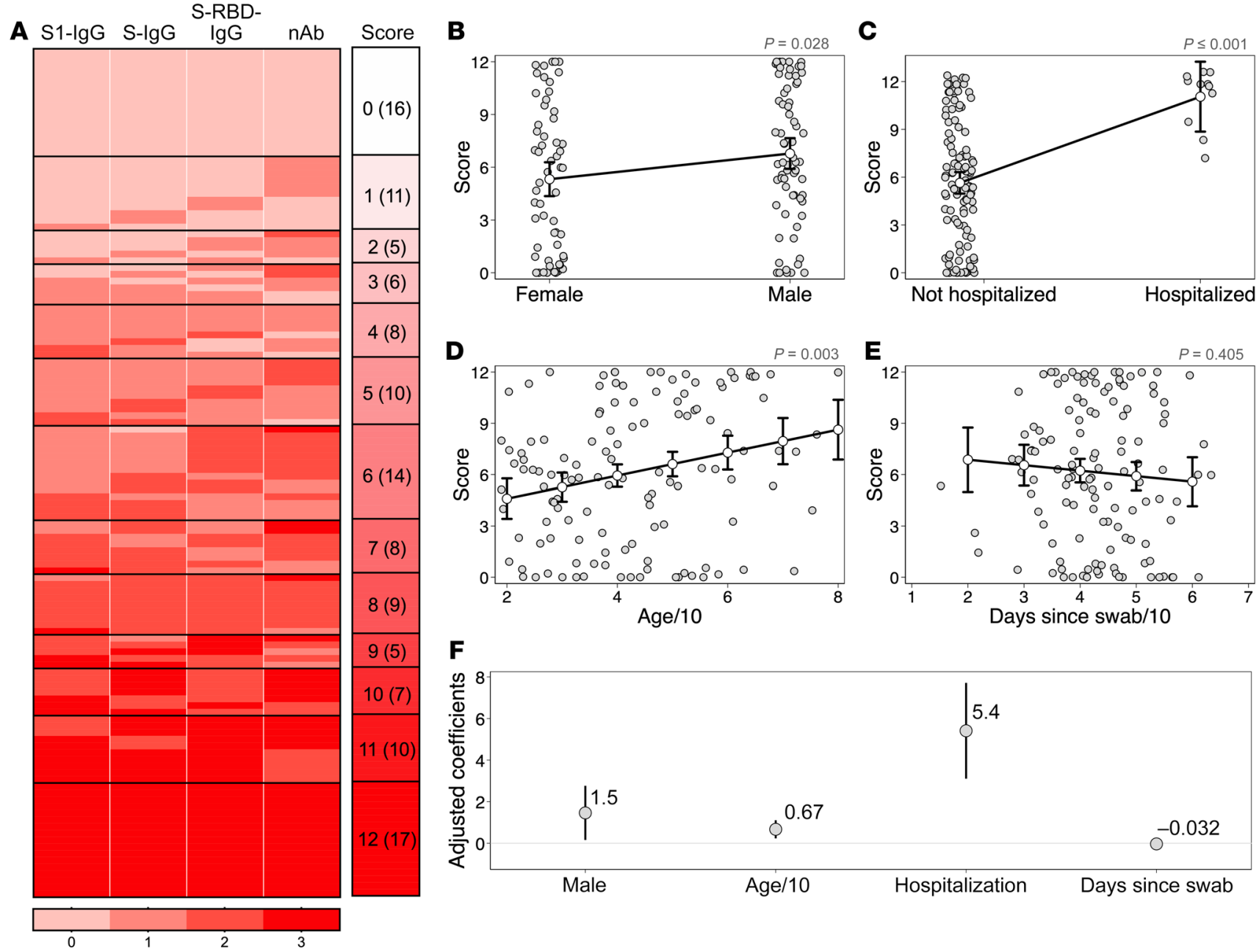

Figure 4. Male sex and hospitalization are predictors of overall greater antibody titers in convalescent plasma. (A) Composite scores were computed for each subject on the basis of the quartile of their response across the anti-S protein domain S1-IgG, anti-S-IgG, anti-S-RBD-IgG, and nAb (NT) assays. The distribution of scores among the study population is shown to the right of the heatmap. (B-E) Multiple linear regression analysis was performed on the continuous outcome of score, including parameters for sex, age in decades, hospitalization status, and number of days since collection of the PCR ${ }^{+}$nasal swab scaled by 10 . For each predictor, the raw data are shown in gray, and the marginal effect plus $95 \% \mathrm{Cl}$ of the given predictor for the average individual in the study is shown in white. $P$ values on top of each panel represent the significance level for the parameter. (F) Summary of the model, where the position of the marker indicates the coefficient value and $95 \% \mathrm{Cl}$ or the expected increase in score for a 1-unit increase in each predictor.

\section{Discussion}

CCP has emerged as a leading therapy for hospitalized COVID-19 patients, with thousands of units collected and more than 30,000 patients treated to date $(5,17)$. There is a compelling argument for why it could be effective either as prophylaxis after exposure, or as treatment for early disease (17). Consequently, it is important to measure the antibody response following recovery from infection with SARS-CoV-2 to understand the characteristics for ideal convalescent plasma donation. These data suggest that diverse antibody isotypes are detectable in plasma approximately 40 days after a positive PCR test for SARS-CoV-2, and IgG is the prominent isotype across diverse assays and analyses. Although the commercial ELISA to detect S1 protein and ELISAs to detect S and S-RBD correlated well with the positive $\mathrm{nAb}$ responses, they were not accurate for confirming samples that were negative for $\mathrm{nAb}$ responses. In addition, although overall antibody levels seemed constant, we noted a significant decrease in nAbs over time. Overall, higher nAb and IgG titers were associated with male sex, older age, and a history of hospitalization, but further investigation is needed to determine whether common or divergent factors are driving these associations.

The heterogeneity in the antibody response demonstrated in this study is consistent with previously published data. While reports from China suggest that the majority of individuals generate higher titers of antibodies 14 or more days after resolution of symptoms (18), 30\% of patients do not appear to develop sufficient $\mathrm{nAb}$ titers following infection (19). The antibody response induced by coronavirus infection in humans tends to be linked to the severity of the disease. Coronaviruses associated with mild disease (e.g., HCoV-229E NL63, OC43, and HKU1) induce transient levels of antibody, whereas those causing more severe disease (e.g., SARS$\mathrm{CoV}$ and MERS-CoV) induce stronger and more durable antibody responses (20). Because SARS-CoV-2 infection spans the spectrum of disease, from asymptomatic to lethal, it is not surprising that the induced antibody responses are heterogeneous. 
In the present study, $20 \%$ of individuals did not have detectable nAbs. Male sex, advancing age, and hospitalization for severe COVID-19 were associated with greater nAb and IgG responses to SARS-CoV-2. Higher IgG titers were correlated with worse COVID-19 outcomes, which is also reflected in the link between greater antibody titers and older age (21). Male sex is also associated with a greater risk of more severe COVID-19 outcomes (22). Greater antibody responses in convalescent plasma from males compared with that from females have been reported (23) and are striking, given that females usually mount stronger immune responses than do males (24). One possible explanation for this apparent reversal in sex-related differences in antibody responses to SARS-CoV-2 is that males with COVID-19 tend to have more severe disease than females and that enhanced inflammatory responses associated with increased disease severity could drive higher B cell recruitment and, consequently, more antibody production. In this regard, the magnitude of antibody responses also correlates with disease severity in other infectious diseases, such as active tuberculosis (25).

There are limitations associated with this study. The samples were cross-sectional, with a relatively tight window of time for data collection. Therefore, the kinetics of the complete antibody response over time could not be determined, and it was difficult to assess how the time relative to the initial diagnosis correlated with the overall titer. The sampled population, however, represented a clinically diverse one, with a wide age range that was representative of the blood donor population. The study was also limited by the lack of nondirect measurements of antibody function (e.g., phagocytosis, antibody-dependent cellular cytotoxicity), but the importance of these mechanisms is not known. Finally, the study focused on antibody responses in plasma, but SARS-CoV-2 antibody responses in the respiratory tract may be critical mediators of protection from infection or severe disease. Understanding the relative contributions of $\operatorname{IgG}, \operatorname{IgM}$, and $\operatorname{IgA}$ to SARS-CoV-2 neutralization will provide insights into the nature of protective antibody responses (26).

Initially, the FDA recommended that convalescent plasma donors would optimally have ELISA titers exceeding 1:320; this was subsequently lowered, given concerns that insufficient numbers of donors would attain this threshold (17). Prior to the emergency use authorization (EUA), the FDA recommended a nAb titer (NT) concentration of 160 or higher, yet allows for a lower titer (1:80) if an alternative is unavailable (27). Under the EUA, this has been revised, whereby units are segregated by low and high titer. Data from the Expanded Access Program and clinical trials are urgently needed to interpret the titers with respect to clinical outcomes and prevention. These results provide a roadmap to select individuals who are likely to have high levels of neutralizing and anti-SARSCoV-2 IgG Abs as preferred convalescent plasma donors.

\section{Methods}

\section{Study participants, blood sample processing, and storage}

Individuals with a history of COVID-19 who were interested in donating convalescent plasma were contacted by study personnel. All subjects had to be at least 18 years of age and have had a confirmed diagnosis of SARS-CoV-2 by detectable RNA on a nasopharyngeal swab. Donors were informed that they needed to satisfy standard eligibility criteria for blood donation (e.g., not pregnant within the previous 6 weeks, never been diagnosed with or have risk factors for transfusion-transmitted infections such as HIV, hepatitis B virus, or hepatitis $C$ virus). These individuals were then invited to participate in the study. Basic demographic information (age, sex, and hospitalization for COVID-19) was obtained from each subject (i.e., potential donor), and confirmation of the original diagnosis of SARS-CoV-2 was required either by medical chart review or sharing of source documentation, including the date the swab was collected and diagnosis was ascertained. Participants were asked the date of symptom onset, the date the positive swab result was reported, and the date of symptom resolution. Approximately $25 \mathrm{~mL}$ whole blood was collected in acidcitrate-dextrose (ACD) tubes. The samples were separated into plasma and PBMCs within 12 hours of collection. The plasma samples were immediately frozen at $-80^{\circ} \mathrm{C}$.

\section{Plasmid preparation}

Recombinant plasmid constructs containing modified S protein or S-RBD and a $\beta$-lactamase (amp) gene were obtained (28) and amplified in E. coli after transformation and growth on Luria broth (LB) agar plates coated with ampicillin. The plasmids were extracted using GigaPrep kits (Thermo Fisher Scientific) and eluted in molecular biology-grade water.

\section{Recombinant protein expression}

HEK293.2sus cells (American Type Culture Collection [ATCC]) were obtained and adapted to Freestyle F-17 medium (Thermo Fisher Scientific) and BalanCD (Irvine Scientific) using polycarbonate shake flasks (Fisherbrand, Thermo Fisher Scientific) with 4 mM GlutaMAX supplementation (Thermo Fisher Scientific). The cells were routinely maintained every 4 days at a seeding density of 0.5 million cells/ $\mathrm{mL}$. The cells in BalanCD were cultured at $37^{\circ} \mathrm{C}$ in $90 \%$ humidity with $5 \% \mathrm{CO}_{2}$, whereas those in $\mathrm{F}-17$ were maintained at $8 \% \mathrm{CO}_{2}$. The cells were counted using the trypan blue dye (Gibco, Thermo Fisher Scientific) exclusion method and a hemocytometer. Cell viability was always maintained above $90 \%$. Twenty-four hours before transfection (day -1), the cells were seeded at a density of 1 million cells/ $\mathrm{mL}$, ensuring that the cell viability was above $90 \%$. Polyethylenimine (PEI) stocks, with a molecular weight of $25 \mathrm{kDa}$ (Polysciences), were prepared in MilliQ water at a concentration of $1 \mathrm{mg} / \mathrm{mL}$. This was sterile filtered through a $0.22 \mu \mathrm{m}$ syringe filter (Corning), aliquoted, and stored at $-20^{\circ} \mathrm{C}$.

On the day of transfection (day 0), the cells were counted to ensure sufficient growth and viability. OptiPRO SFM (Gibco, Thermo Fisher Scientific) was used as the medium for the transfection mixture. For 100 $\mathrm{mL}$ cell culture, 2 tubes were aliquoted with $6.7 \mathrm{~mL}$ each of OptiPRO, one for PEI and the other for rDNA. A DNA/PEI ratio of 1:3.5 was used for transfection. A volume of $350 \mu \mathrm{L}$ prepared PEI stock solution was added to tube 1 , while $100 \mu \mathrm{g}$ rDNA was added to tube 2 and incubated for 5 minutes. Following incubation, these were mixed together, incubated for 10 minutes at room temperature, and then added to the culture through gravity addition. The cells were then returned to the $37^{\circ} \mathrm{C}$ incubator. One day after transfection (day 1), the cells were spun down at $400 \mathrm{~g}$ for 7 minutes at room temperature and resuspended in fresh media with GlutaMAX supplementation. Three to 5 hours after resuspension, 0.22 $\mu \mathrm{m}$ sterile, filtered sodium butyrate (MilliporeSigma) was added to the flask at a final concentration of $5 \mathrm{mM}$. The cells were allowed to grow 
for a period of 4-5 days. Cells were counted and their viability assessed, and glucose and lactate values were measured every day. Cells were harvested when either the viability fell below $60 \%$ or when the glucose was depleted, followed by centrifugation at $900 \mathrm{~g}$ for 10 minutes at room temperature. Cell culture supernatants containing either recombinant $\mathrm{RBD}$ or S protein were filtered through $0.22 \mu \mathrm{m}$ polyethersulfone (PES) membrane stericup filters (Millipore MilliporeSigma) to remove cell debris and then stored at $-20^{\circ} \mathrm{C}$ until purification.

\section{Protein purification}

Protein purification by immobilized metal affinity chromatography (IMAC) and gravity flow was adapted from previous methods (28). After washing with PBS (Thermo Fisher Scientific), nickel nitrilotriacetic acid (Ni-NTA) agarose (QIAGEN) was added to the culture supernatant, followed by overnight incubation for $12-16$ hours at $4^{\circ} \mathrm{C}$ on a rotator. For every $150 \mathrm{~mL}$ of culture supernatant, $2.5 \mathrm{~mL} \mathrm{Ni-NTA}$ agarose was added. Five-milliliter gravity-flow polypropylene columns (QIAGEN) were equilibrated with PBS. One polypropylene column was used for every $150 \mathrm{~mL}$ culture supernatant. The supernatant-agarose mixture was then loaded onto the column to retain the agarose beads, with recombinant proteins bound to the beads. Each column was then washed, first with $1 \times$ culture supernatant volume of PBS and then with $25 \mathrm{~mL}$ of $20 \mathrm{mM}$ imidazole (Millipore MilliporeSigma) in PBS wash buffer to remove host cell proteins. Recombinant proteins were then eluted from each column in 3 fractions with $5 \mathrm{~mL}$ of $250 \mathrm{mM}$ imidazole in PBS elution buffer per fraction, giving a total of $15 \mathrm{~mL}$ eluate per column. The eluate was subsequently dialyzed several times against PBS using Amicon Ultra Centrifugal Filters (Millipore MilliporeSigma) at $5000 \mathrm{~g}$ for 20 minutes at $10^{\circ} \mathrm{C}$ to remove the imidazole and concentrate the eluate. Filters with a $10 \mathrm{kDa}$ molecular weight cutoff were used for RBD eluate, whereas filters with a $50 \mathrm{kDa}$ molecular weight cutoff were used for full-length $S$ protein eluate. The final concentration of the recombinant RBD and $S$ proteins was measured by bicinchoninic acid (BCA) assay (Thermo Fisher Scientific), and purity was assessed on 10\% SDS-PAGE gels (Bio-Rad) followed by Coomassie blue staining. After sufficient destaining in water overnight, clear single bands were visible for RBD and S proteins at their respective molecular sizes.

\section{Viruses and cells}

Vero-E6 cells (CRL-1586, ATCC) and Vero-E6-TMPRSS2 cells (29) were cultured in complete media (CM) consisting of DMEM containing $10 \%$ FBS (Gibco, Thermo Fisher Scientific), $1 \mathrm{mM}$ glutamine (Invitrogen, Thermo Fisher Scientific), $1 \mathrm{mM}$ sodium pyruvate (Invitrogen, Thermo Fisher Scientific), $100 \mathrm{U} / \mathrm{mL}$ penicillin (Invitrogen, Thermo Fisher Scientific), and $100 \mu \mathrm{g} / \mathrm{mL}$ streptomycin (Invitrogen, Thermo Fisher Scientific). Cells were incubated in a $5 \% \mathrm{CO}_{2}$ humidified incubator at $37^{\circ} \mathrm{C}$.

The SARS-CoV-2/USA-WA1/2020 virus was obtained from BEI Resources. The infectious virus titer was determined on Vero cells using a $50 \%$ tissue culture infectious dose $\left(\mathrm{TCID}_{50}\right)$ assay as previously described for SARS-CoV $(30,31)$. Serial 10-fold dilutions of the virus stock were made in infection media (IM) (identical to CM except the FBS was reduced to $2.5 \%$ ), and then $100 \mu \mathrm{L}$ of each dilution was added to the Vero cells in a 96-well plate in sextuplicate. The cells were incubated at $37^{\circ} \mathrm{C}$ for 4 days, visualized by staining with naphthol blue-black, and scored visually for cytopathic effect. A Reed and Muench calculation was used to determine the $\mathrm{TCID}_{50}$ per $\mathrm{mL}$ (32).

\section{ELISAs}

Commercial ELISAs and avidity. The EUROIMMUN Anti-SARSCoV-2 ELISA for both IgA (catalog EI2606-9601A) and IgG (catalog EI2606-9601G) was validated in a Clinical Laboratory Improvement Amendments-certified (CLIA-certified) laboratory. The assay was performed according to the manufacturer's specifications. The OD of the sample divided by the OD of the calibrator from that run and the ratio constitute the AU. According to the manufacturer, an AU of 0-0.79 is considered negative, $0.80-0.99$ is borderline, and 1.0 or higher is positive.

To measure anti-SARS-CoV-2 IgG avidity, each plate had the following components: $100 \mu \mathrm{L}$ plasma (1:101 dilution, per the manufacturer's protocol) and $100 \mu \mathrm{L}$ undiluted positive, negative, and calibrator controls. Plates containing reaction components were incubated for 1 hour at $37^{\circ} \mathrm{C}$ followed by 3 washes. A $300 \mu \mathrm{L}$ volume of wash buffer containing urea at varying concentrations $(0 \mathrm{M}, 1 \mathrm{M}, 2 \mathrm{M}$, $4 \mathrm{M}, 6 \mathrm{M}$, or $8 \mathrm{M}$ ) was added to the plates and incubated at $37^{\circ} \mathrm{C}$ for 10 minutes (33). Plates were washed 3 times, followed by the manufacturer's protocol for addition of conjugate and substrate. Ratios of 0.8 or higher were considered positive. Fifty percent $K_{D}$ calculations were performed with an AAT Bioquest $\mathrm{IC}_{50}$ calculator using a 4-parameter logistic regression model. The Quest Graph $\mathrm{IC}_{50}$ Calculator was retrieved from https://www.aatbio.com/tools/ic50-calculator.

Indirect ELISAs. The ELISA protocol was adapted from a protocol published by the Florian Krammer laboratory (28). Ninety-sixwell plates (Immulon 4HBX, Thermo Fisher Scientific) were coated with either full-length S protein or S-RBD at a volume of $50 \mu \mathrm{L}$ of 2 $\mu \mathrm{g} / \mathrm{mL}$ diluted antigen in filtered, sterile $1 \times$ PBS (Thermo Fisher Scientific) at $4^{\circ} \mathrm{C}$ overnight. Coating buffer was removed and the plates were washed 3 times with $300 \mu \mathrm{L} 1 \times$ PBS plus $0.1 \%$ Tween-20 (PBST) wash buffer (Thermo Fisher Scientific) and then blocked with $200 \mu \mathrm{L}$ PBST with 3\% nonfat milk (milk powder, American Bio) by volume for 1 hour at room temperature. All plasma samples were heat inactivated at $56^{\circ} \mathrm{C}$ on a heating block for 1 hour before use. Negative control samples were prepared at 1:10 dilutions in PBST in 1\% nonfat milk and plated at a final concentration of 1:100. A mAb against the SARSCoV-2S protein was used as a positive control (1:5000; catalog 40150D001, Sino Biological). For serial dilutions of plasma on either S- or S-RBD-coated plates, plasma samples were prepared in 3-fold serial dilutions starting at 1:20 in PBST in 1\% nonfat milk. Blocking solution was removed, and $10 \mu \mathrm{L}$ diluted plasma was added in duplicate to the plates and incubated at room temperature for 2 hours. Plates were washed 3 times with PBST wash buffer, and $50 \mu \mathrm{L}$ secondary antibody was added to the plates and incubated at room temperature for 1 hour. The following anti-human secondary antibodies were used: Fc-specific total IgG HRP (1:5000 dilution, catalog A18823, Invitrogen, Thermo Fisher Scientific), IgM heavy-chain HRP (1:5000; catalog A18835, Invitrogen, Thermo Fisher Scientific), and IgA cross-adsorbed HRP (1:5000; catalog A18787, Invitrogen, Thermo Fisher Scientific). All antibodies were prepared in PBST plus $1 \%$ nonfat milk. Plates were washed and all residual liquid removed before addition of $100 \mu \mathrm{L}$ SIGMAFAST OPD ( $o$-phenylenediamine dihydrochloride) solution (MilliporeSigma) to each well, followed by incubation in darkness at room temperature for 10 minutes. To stop the reaction, $50 \mu \mathrm{L} 3 \mathrm{M} \mathrm{HCl}$ (Thermo Fisher Scientific) was added to each well. The OD of each plate was read at $490 \mathrm{~nm}\left(\mathrm{OD}_{490}\right)$ on a SpectraMax i3 ELISA Plate Reader (BioTek Instruments). The positive cutoff value for each plate 
was calculated by summing the average of the negative values and 3 times the SD of the negatives. All values at or above the cutoff value were considered positive.

\section{Microneutralization assay}

Plasma nAbs were determined as described for SARS-CoV (34). Twofold dilutions of plasma (starting at a 1:20 dilution) were made in IM. Infectious virus was added to the plasma dilutions at a final concentration of $1 \times 10^{4} \mathrm{TCID}_{50} / \mathrm{mL}\left(100 \mathrm{TCID}_{50}\right.$ per $\left.100 \mu \mathrm{L}\right)$. The samples were incubated for 1 hour at room temperature, and then $100 \mu \mathrm{L}$ of each dilution was added to 1 well of a 96-well plate of VeroE6-TMPRSS2 cells in sextuplet for 6 hours at $37^{\circ} \mathrm{C}$. The inocula were removed, fresh IM was added, and the plates were incubated at $37^{\circ} \mathrm{C}$ for 2 days. The cells were fixed by the addition of $150 \mu \mathrm{L}$ of $4 \%$ formaldehyde per well, incubated for at least 4 hours at room temperature, and then stained with Napthol Blue Black (MilliporeSigma). The nAb titer was calculated as the highest serum dilution that eliminated the cytopathic effect in $50 \%$ of the wells.

\section{Statistics}

Descriptive analyses. AUC values were computed by plotting normalized OD values against sample dilution for ELISAs. The AUC for micronentralization assays used the exact number of wells protected from infection at each plasma dilution. For each assay, samples with titers below the limit of detection were assigned an arbitrary AUC value of half of the lowest measured AUC value. The data were then log transformed to achieve a normal distribution. Descriptive statistics stratified by sex are presented as medians and IQRs, and male versus female comparisons overall and in each age category were done using $t$ tests. A $P$ value of less than 0.05 was considered statistically significant. AUC values for IgG, IgA, and IgM were compared by 1-way ANOVA. Correlations between antibody isotypes and assays were assessed using Pearson's correlation coefficient. Where binary cutoffs were available, IgG data were dichotomized using the 1:320 cutoff originally recommended by the FDA (17) or the cutoff of AU greater than 0.8 suggested by the manufacturer. The association between ELISA and microneutralization results were then calculated using nAb titers (i.e., titer $>1: 20$ ) as the reference.

Predictors of assay-specific responses. Multiple linear regression models were used to assess the impact of the demographic (age in decades and sex) and clinical factors (hospitalization status and days since collection of the $\mathrm{PCR}^{+}$swab scaled by 10) on S1-IgG OD values, $\log$ AUC values for anti-RBD and anti-spike IgG, as well as nAbs. The 4 time-related terms collected from the participants (i.e., date of symptom onset, date the $\mathrm{PCR}^{+}$swab was collected, date that the positive swab result was reported, and date of symptom resolution) were correlated with each other. To avoid collinearity, only the number of days since collection of the $\mathrm{PCR}^{+}$swab was included in analyses, as this was the only metric that was not subject to response and recall bias and therefore deemed the most reliable. All predictor estimates were adjusted for the 3 other parameters in the model. Various additional parameters were tested, including an interaction term between age and sex and linear splines at different ages, but these decreased the overall fit of the model and were therefore not included in further analyses. Data are presented as the marginal effect of each predictor for the average person in the study population (35) along with coefficients and $95 \%$ CIs of each estimate.
Composite score representing the overall quality of the antibody response. Composite scores were computed to provide a single metric as a proxy for the overall quality of the antibody response. The responses for S1-IgG, S-IgG, S-RBD, and neutralizing assays were divided into quartiles, and subjects were assigned a score of 0 (lowest) to 3 (highest) quartile for each assay. The assay-specific scores were summed to create the composite score, ranging from 0 (lowest quartile for each assay) to 12 (highest quartile for each assay). A multiple linear regression model was then performed on the composite score, including parameters for sex, age in decades, hospitalization status, and number of days since collection of the $\mathrm{PCR}^{+}$swab (scaled by 10). As above, data are presented as the marginal effect of each predictor for the average person in the study population (35) along with coefficients and 95\% CIs of each estimate. All analyses were performed using GraphPad Prism 8 (GraphPad Software) and Stata 15 (StataCorp).

\section{Study approval}

The Johns Hopkins University School of Medicine IRB reviewed and approved the sample collection protocols and overall study. All participating subjects provided written informed consent.

\section{Author contributions}

EMB, AC, and AART conceived and designed the study. EMB, DS, SS, and AART wrote the IRB protocol. RS, AAW, RMH, IB, EMB, and AART recruited participants. SLK, AP, HSP, RLU, KL, OL, TCQ, SEB, ADR, PC, EMB, and AART carried out all experiments. $\mathrm{HMN}$, SK, and MJB produced recombinant SARS-CoV-2 proteins. JRS performed statistical analyses. SLK, AP, HSP, RLU, JRS, EMB, $\mathrm{AC}$, and AART wrote the manuscript with substantial input from all co-authors.

\section{Acknowledgments}

We are grateful to all the study participants who donated plasma, the clinical staff, including Sonali Thapa, Liz Martinez, Mary De'Jarnette, Carlos Aguado, Peggy Iraola, and Jackie Lobien, who collected samples, and the technical staff, including Yolanda Eby, Rey Fernandez, Haley Schmidt, Charles Kirby, Ethan Klock, Owen Baker, Jernelle Miller, and Morgan Keruly, who aliquoted and stored samples for this study. We thank Florian Krammer of the Icahn School of Medicine at Mount Sinai for providing protocols, plasmids, and initial stocks of ELISA antigens and Daniel Smith for assistance with the graphical abstract generated in BioRender. We thank the National Institute of Infectious Diseases, Japan, for providing VeroE6TMPRSS2 cells and acknowledge the Centers for Disease Control and Prevention, BEI Resources, NIAID, NIH for SARS-related coronavirus 2, isolate USAWA1/2020, NR-5228. This work was supported in part by grants from the NIH Specialized Center of Research Excellence (U54AG062333, to SLK, AP, HSP, and JRS); the NIH Center of Excellence in Influenza Research and Surveillance (HHSN272201400007C, to $\mathrm{AP}, \mathrm{KL}, \mathrm{SLK}$, and RLU); the NIH Molecular and Cellular Basis of Infectious Diseases program (T32A1007417, to RLU); the NIAID (AI052733 and AI15207, to AC, and R01AI120938, R01AI120938S1, and R01AI128779, to AART); the Division of Intramural Research, NIAID (to OL and TQ); the National Heart, Lung and Blood Institute (NHLBI) (1K23HL151826-01, to EBM, and R01HL059842, to AC); Bloomberg Philanthropies (to AC); and the US Department of Defense (W911QY2090012, to AC and DS). 
Address correspondence to: Sabra L. Klein, W. Harry Feinstone Department of Molecular Microbiology and Immunology, Bloomberg School of Public Health, Johns Hopkins University, 615 N. Wolfe Street, Baltimore, Maryland 21205, USA. Email: sklein2@jhu.edu. Or to: Aaron A.R. Tobian, Department of Pathology, School of Medicine, Johns Hopkins University, 600 N. Wolfe Street, Carnegie Room 437, Baltimore, Maryland 21287, USA.Email: atobian1@jhmi.edu.
1. JHU. Johns Hopkins University Coronavirus Resource Center. https://coronavirus.jhu.edu/ map.html. Accessed 10/6/2020.

2. Casadevall A, Pirofski LA. The convalescent sera option for containing COVID-19. JClin Invest. 2020;130(4):1545-1548.

3. Shen C, et al. Treatment of 5 critically ill patients with COVID-19 with convalescent plasma. JAMA. 2020;323(16):1582-1589.

4. Duan K, et al. Effectiveness of convalescent plasma therapy in severe COVID-19 patients. Proc Natl Acad Sci U S A . 2020;117(17):9490-9496.

5. Joyner MJ, et al. Early safety indicators of COVID-19 convalescent plasma in 5000 patients. JClin Invest. 2020;130(9):4791-4797.

6. Li L, et al. Effect of convalescent plasma therapy on time to clinical improvement in patients with severe and life-threatening COVID-19: a randomized clinical trial. JAMA. 2020;324(5):1-11.

7. Liu STH, et al. Convalescent plasma treatment of severe COVID-19: a matched control study. medRxiv. https://doi.org/10.1101/2020. 05.2.20102236. Published May 22, 2020. Accessed September 3, 2020.

8. Hegerova L, et al. Use of convalescent plasma in hospitalized patients with COVID-19: case series. Blood. 2020;136(6):759-762.

9. Gharbharan A, et al. Convalescent plasma for COVID-19. A randomized clinical trial. medRxiv. https://doi.org/10.1101/2020.07.01.2013985. Published July 3, 2020. Accessed September 3, 2020.

10. Zost SJ, et al. Potently neutralizing and protective human antibodies against SARS-CoV-2. Nature. 2020;584(7821):443-449.

11. Walls AC, Park YJ, Tortorici MA, Wall A, McGuire AT, Veesler D. Structure, function, and antigenicity of the SARS-CoV-2 spike glycoprotein. Cell. 2020;181(2):281-292.

12. Wang $Q$, et al. Structural and functional basis of SARS-CoV-2 entry by using human ACE2. Cell. 2020;181(4):894-904 e9.

13. Shang J, et al. Cell entry mechanisms of SARS-CoV-2. Proc Natl Acad Sci U S A. 2020;117(21):11727-11734.

14. VanBlargan LA, Goo L, Pierson TC. Deconstructing the antiviral neutralizing-antibody response: implications for vaccine development and immunity. Microbiol Mol Biol Rev. 2016;80(4):989-1010.

15. Klasse PJ. Neutralization of virus infectivity by antibodies: old problems in new perspectives. Adv Biol. 2014;2014:157895.

16. Vanderven HA, Kent SJ. The protective potential of Fc-mediated antibody functions against influenza virus and other viral pathogens. Immunol Cell Biol. 2020;98(4):253-263.

17. Bloch EM, et al. Deployment of convalescent plasma for the prevention and treatment of COVID-19. J Clin Invest. 2020;130(6):2757-2765.

18. Duan K, et al. The feasibility of convalescent plasma therapy in severe COVID-19 patients: a pilot study. medRxiv. https://doi.org/10.1101/20 20.03.16.20036145. Published March 23, 2020. Accessed September 3, 2020.

19. Wang $X$, et al. Neutralizing antibodies responses to SARS-CoV-2 in COVID-19 inpatients and convalescent patients. medRxiv. https://doi.org/10. 1101/2020.04.15.20065623. Accessed April 23, 2020. Accessed September 3, 2020.

20. Sariol A, Perlman S. Lessons for COVID-19 immunity from other coronavirus infections. Immunity. 2020;53(2):248-263.

21. Zhang B, et al. Immune phenotyping based on neutrophil-to-lymphocyte ratio and IgG predicts disease severity and outcome for patients with COVID-19. medRxiv. https://doi.org/10.1101/20 20.03.12.20035048. Published March 16, 2020. Accessed September 3, 2020.

22. Scully EP, Haverfield J, Ursin RL, Tannenbaum C, Klein SL. Considering how biological sex impacts immune responses and COVID-19 outcomes. Nat Rev Immunol. 2020;20(7):442-447.

23. Robbiani DF, et al. Convergent antibody responses to SARS-CoV-2 in convalescent individuals. Nature. 2020;584(7821):437-442.

24. Flanagan KL, Fink AL, Plebanski M, Klein SL. Sex and gender differences in the outcomes of vaccination over the life course. Annu Rev Cell Dev Biol. 2017;33:577-599.

25. Yu X, Prados-Rosales R, Jenny-Avital ER, Sosa K, Casadevall A, Achkar JM. Comparative evaluation of profiles of antibodies to mycobacterial capsular polysaccharides in tuberculosis patients and controls stratified by HIV status. Clin Vaccine Immunol. 2012;19(2):198-208.

26. Sterlin D, et al. IgA dominates the early neutralizing antibody response to SARS-CoV-2. medRxiv. https://doi.org/10.1101/2020.06.10. 20126532. Published June 11, 2020. Accessed September 3, 2020.

27. [No authors listed]. Recommendations for Investigational COVID-19 Convalescent Plasma. Food and Drug Administration. https:// www.fda.gov/vaccines-blood-biologics/ investigational-new-drug-ind-or-deviceexemption-ide-process-cber/recommendationsinvestigational-covid-19-convalescentplasma. Updated September 2, 2020. Accessed September 3, 2020.

28. Stadlbauer D, et al. SARS-CoV-2 seroconversion in humans: a detailed protocol for a serological assay, antigen production, and test setup. Curr Protoc Microbiol. 2020;57(1):e100.

29. Matsuyama S, et al. Enhanced isolation of SARSCoV-2 by TMPRSS2-expressing cells. Proc Natl Acad Sci U S A. 2020;117(13):7001-7003.

30. Schaecher SR, Touchette E, Schriewer J, Buller RM, Pekosz A. Severe acute respiratory syndrome coronavirus gene 7 products contribute to virus-induced apoptosis. J Virol. 2007;81(20):11054-11068.

31. Schaecher SR, Mackenzie JM, Pekosz A. The ORF7b protein of severe acute respiratory syndrome coronavirus (SARS-CoV) is expressed in virus-infected cells and incorporated into SARSCoV particles. J Virol. 2007;81(2):718-731.

32. Reed LJ, Meunch H. A simple method of estimating 50 percent endpoints. Am J Epidemiol. 1938;27(3):493-497.

33. Wang $\mathrm{Q}$, et al. A method to prevent SARS-CoV-2 IgM false positives in gold immunochromatography and enzyme-linked immunosorbent assays. JClin Microbiol. 2020;58(6):e00375-20.

34. Schaecher SR, et al. An immunosuppressed Syrian golden hamster model for SARS-CoV infection. Virology. 2008;380(2):312-321.

35. Williams R. Using the margins command to estimate and interpret adjusted predictions and marginal effects. Stata J. 2012;12(2):308-331. 